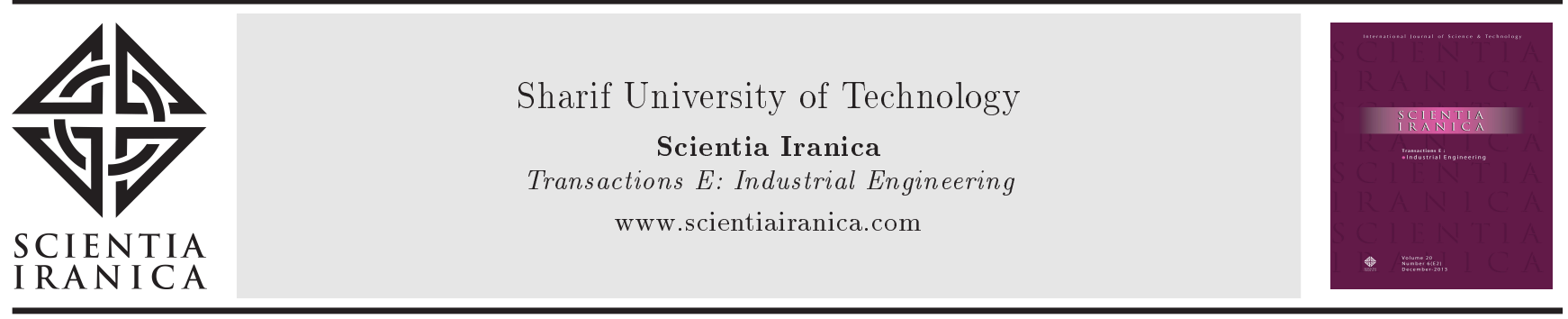

\title{
Economic design of cumulative count of conforming control charts based on average number of inspected items
}

\author{
M.S. Fallahnezhad* and V. Golbafian \\ Department of Industrial Engineering, Yazd University, Yazd, P.O. Box 89195-741, Iran. \\ Received 17 February 2015; received in revised form 5 October 2015; accepted 2 February 2016
}

\author{
KEYWORDS \\ Statistical process \\ control; \\ High quality \\ processes; \\ Cumulative count of \\ conforming control \\ charts; \\ Average number of \\ inspected items; \\ Economic design of \\ control charts.
}

\begin{abstract}
Nowadays, continuous improvement can be regarded as the essence of survival and growth in order to not only increase the competition in global market, but also requirement for ever-decreasing defect levels in processes. Therefore, new statistical analysis techniques and decision-making procedures have been continuously evolved, both to handle high quality processes and to look for process improvement opportunities. CCC- $r$ chart, or extended approach of CCC charts, is generally a technique for high quality processes, when nonconforming items are rarely observed. This study develops a mathematical model based on the average number of inspected items for the economic design of CCC- $r$ chart, so that the average cost per item is minimized. The optimal designed parameters for different nonconforming fractions and different parameters in each iteration are calculated. In addition, with respect to Type I error $(\alpha)$ and Type II error $(\beta)$ in the process, sensitivity analysis of the model is carried out.

(C) 2017 Sharif University of Technology. All rights reserved.
\end{abstract}

\section{Introduction}

Control charts, the most important Statistical Process control tools, are effective control techniques that explicitly monitor process characteristics in order to maintain the process statistically controlled; using the statistical process control enables users to estimate the process parameters.

High-quality or high-yield processes refer to those production or transactional processes that are capable of producing very low levels of non-conforming fraction. Zhang et al. [1] emphasized that modern manufacturing industries are high-quality processes because of various efforts such as wide automation and deployment of effective quality management methodologies or strate-

*. Corresponding author. Tel.: +983531232548;

Fax: +9835 38210699

E-mail addresses: Fallahnezhad@yazd.ac.ir (M.S.

Fallahnezhad); V.golbafian.90@gmail.com (V. Golbafian) gies. Bersimis et al. [2] expressed that if a small or moderate shift occurs in these processes, then the outof-control non-conforming fraction items will still be very small; therefore, it is highly probable that no defective item will be observed in the inspected sample. Therefore, for small or moderate shifts, standard $p$ or $n p$ charts fail to diagnose a change in a high-quality process.

The idea behind the CCC (Cumulative Count of Conforming) control chart, first developed by Calvin [3] to monitor high-quality processes, is based on geometric distribution; the number of conforming items between two consecutive nonconforming ones changes when the nonconforming fraction shifts. Khilare and Shirka [4] argued about m-of-m control chart based on cumulative count of conforming units for high-yield processes. They compared performance of the m-of-m control chart with control chart based on cumulative count of conforming units. Chen et al. [5] presented a CCC chart with variable sampling 
intervals and control limits. Amiri and Khosravi [6] provided a maximum likelihood estimator for the change point of the nonconforming level in the highquality process with a linear trend. Ahmadi and Fallahnezhad $[7,8]$ presented an acceptance-sampling plan based on cumulative count of conforming using minimum angle method. In addition, they compared count of cumulative conforming sampling plans with Dodge-Romig single sampling plan. Their method had better performance in most of the cases. AcostaMejia [9] explained that since the geometric probability function is highly asymmetric; thus, this chart is not very sensitive to signaling small to moderate increases in the nonconforming proportion, $p$.

An alternative strategy to rising sensitivity of charts is to monitor the cumulative count of items produced until a fixed number " $r$ " of nonconforming items are observed. A chart on this basis, called CCC$r$ chart, has a better performance in finding the shifts of nonconforming proportion in high-quality processes. It is well known that this number will follow the Negative Binomial distribution, which is a generalization of the Geometric distribution. The idea of CCC- $r$ control chart was proposed by Xie et al. [10].

For the efficient use of control charts, the appropriate sample size, sampling frequency, and control limits must be selected before the application of the classical control methods. The procedure of selecting these parameters is dependent on the control charts design. For the CCC- $r$ chart, determining $r$ can be considered a subjective issue, and this is similar to the case of sample size in classical ones. By increasing fixed number $r$, sensitivity and capability of charts increase; thus, parameters' determination has a significant effect on charts' efficiency.

By finding essential parameters in chart, the process would be in a controllable and consolidate situation. Yilmaz and Burnak [11] discussed that cost consideration is always important in industry and any related activities should be put into the context of cost saving to improve profitability. To use control chart in practice, economic factors can be taken into account for designing control chart. From the economic perspective of control chart design, costs, such as those related to sampling, testing, investigating out of control signals, eliminating special cases, and sending defective products to the customer, are affected by design of control charts.

Ohta et al. [12] proposed an economic model to determine the optimal parameters of a CCC- $r$ chart: $r$, the sampling interval, and the lower control limit. Chan et al. [13] presented a two-stage CCC- $r$ control scheme inspired by double sampling plans to detect the upward shift of non-conforming fraction. An economic model was then proposed to choose the design parameters at each stage. The Average Number of Inspected items (ANI) is an alternative performance metric for control charts, and it is defined as the expected number of inspected items before the chart signals an alarm. Chen [14] extended a procedure to obtain the control limits with near maximal $\mathrm{ANI}_{0}$ and the near-unbiased $\mathrm{ANI}_{0}$ to use this index as performance criterion for charts and determining control limits. There are numerous studies dedicated to the economic design of control charts. Duncan [15] was the first who studied the economic design of control charts, and Lorenzen and Vance [16] generalized the original model proposed by Duncan and made the model directly applicable to many types of control charts. Lorenzen and Vance's new modeling approach considers whether production continues while special causes are being investigated and the process is being adjusted. Mortarino [17] performed a sensitivity analysis for the cost parameters and analyzed the behavior of optimal solutions of Duncan's model in the X-control chart. Celano et al. [18] investigated the economic design of a Shewhart control chart to monitor the process mean in a short production run. Zhang et al. [19] discussed that the economic design of control chart aims to maximize the profit (or minimizing the cost) associated with implementation of the SPC scheme in a process and presented the economic design of a control chart. Kudo et al. [20] proposed an economic design of a dynamic CCC- $r$ chart with time-varying parameters. They explained a process control method for a Weibull distributed-shock model and determined the initial values and dynamic decision rules for the time-varying parameters of the CCC- $r$ chart, that is, the required number of nonconforming observations $r$, the sampling interval, $h$, and the Lower Control Limit (LCL) maximizing the expected profit per unit time derived from the process. Sherbaf Moghaddam et al. [21] developed a multi-objective model for the economic-statistical design of the CCC control chart. Then, multi-objective evolutionary algorithm (NSGAII) is proposed for obtaining the Pareto optimal solutions. The results showed that the proposed multiobjective model could address the disadvantages of the economic model. In other words, multi-objective economic-statistical design of the CCC control chart is better than that of the economical one.

It is important to design the appropriate control chart in order to prepare high-quality production and maintain the process in an ideal situation with profitable production. The purpose of this model is to present an economic model for a production cycle that minimizes the expected average cost per produced item. The proposed model is studied on two-sided CCC- $r$ chart, and upper and lower control limits can be determined as decision variables separately; most of models in the literature have been proposed for one-sided charts and have only examined lower control 
limit.

Even though Duncan or Lorenzen-Vence objective functions $[15,16]$ are used to optimize the average cost or net profit per unit time in most of previous studies, our proposed objective function will optimize the average cost per "item produced" which is more practical in high-yield processes. Because of the high production rate per unit time in these situations, the average cost is decreased, which is the most important interest of producers. Thus, the study on expected process control costs per produced item is more appropriate due to minimizing expected average cost in a cycle along with high rate of production. In addition, this model specifies the optimal value of $\mathrm{r}$ parameter in different modes of nonconforming proportion, and a sensitivity analysis is on nonconforming fractions in this economic model. To increase the ability of model in process statistical studies, two constraints are considered based on acceptable false alarm probability (Type I error) and observing process as in control, while the process is actually out of control (Type II error). These constraints limit the feasible solution space, so that the decision variables are determined more accurately, and the economic model is modified to an economic-statistical one, which is one of the advantages of the proposed model in high-quality processes. Although ARL is used in most of economic models as performance index. But, for more accurate optimization, ANI, or Average Number of Inspected, item is used as performance measure in this model to determine chart parameters, containing more information about the process because it counts the number of inspected items and is more reliable for high yield processes.

In section two of the paper, the formulation of CCC- $r$ charts and economic model will be reviewed, and statement of problem is discussed completely. In Section 3, a numerical example will be given to explain and analyze the model in practice. Section 4 is the conclusion section.

\section{Problem statement formulation}

\subsection{Review of CCC-r chart}

The idea of CCC chart can be extended to process monitoring by considering the number of inspected items until a fixed number of nonconforming items are observed. One such control chart is called the cumulative count of conforming chart (CCC- $r$ chart). In a CCC- $r$ chart, the number of items until the $r$ th nonconforming item $(x)$ is plotted to monitor $p \in(0,1)$, the nonconforming fraction of the process. Let $r$ be a fixed positive integer, $x$ is then a random variable of negative binomial distribution with the probability mass function (pmf) and the cumulative distribution function (cdf) as follows:

$$
\begin{aligned}
& f_{r, p}(x)=\left(\begin{array}{l}
x-1 \\
r-1
\end{array}\right)(1-p)^{x-r} p^{r} ; \\
& \text { for } x=r, r+1, \ldots \\
& F_{r, p}(n)=p(x \leq n)=\sum_{i=r}^{n}\left(\begin{array}{l}
i-1 \\
r-1
\end{array}\right)(1-p)^{i-r} p^{r} \\
& =1-\sum_{k=0}^{r-1}\left(\begin{array}{l}
x \\
k
\end{array}\right)(1-p)^{x-k} p^{k} ;
\end{aligned}
$$

for $x=r, r+1, \ldots$

When $r$ is equal to 1 , as the case of CCC chart, the distribution reduces to a geometric distribution.

For an acceptable risk of false alarm, $\alpha$, Xie et al. [22] presented the equation for determining the Upper Control Limit, UCL, and the Lower Control Limit, LCL, of the CCC- $r$ chart:

$$
F(\mathrm{UCL}, r, p)=\sum_{i=r}^{\mathrm{UCL}}\left(\begin{array}{l}
i-1 \\
r-1
\end{array}\right) p^{r}(1-p)^{i-r}=1-\frac{\alpha}{2},
$$

and:

$$
F(\mathrm{LCL}, r, p)=\sum_{i=r}^{\mathrm{LCL}-1}\left(\begin{array}{l}
i-1 \\
r-1
\end{array}\right) p^{r}(1-p)^{i-r}=\frac{\alpha}{2},
$$

where the centerline, CL, is given as the solution:

$$
F(\mathrm{CL}, r, p)=\sum_{i=r}^{\mathrm{CL}}\left(\begin{array}{l}
i-1 \\
r-1
\end{array}\right) p^{r}(1-p)^{i-r}=\frac{1}{2} \text {. }
$$

As is clear from the above equations, the control limits of the CCC- $r$ chart will be increased when the process of nonconforming fraction, $p$, approaches zero. The control limits of the CCC- $r$ charts with a large $r$ parameter are much larger than the small $r$ parameter for the same value of $p$.

Researchers often apply Average Run Length (ARL) index to their analysis, which denotes the average number of the sample taken before a process change is detected. Likewise, we can define ARL as average number of points plotted on the chart between two successive alarm signals as discussed by Chan et al. [13] and Chen et al. [14]:

$$
\mathrm{ARL}=\frac{1}{1-p(\mathrm{LCL} \leq x \leq \mathrm{UCL})}=\frac{1}{1-k(p)} .
$$

Let $k(p)=p$ (not having any signal while detecting $r$ th nonconforming item | the fraction nonconforming of the process is $p$ ):

$$
k(p)=\sum_{i=\mathrm{LCL}}^{\mathrm{UCL}}\left(\begin{array}{l}
i-1 \\
r-1
\end{array}\right) p^{r}(1-p)^{i-r}
$$

In the following, we try to determine the optimal value of LCL, UCL, and $r$ parameter with respect 
to economic considerations where LCL and UCL are the lower and upper control limits, respectively. Concerning Chen et al. [14], ANI can be approximately calculated as:

$$
\mathrm{ANI}=\frac{r}{p} \cdot \mathrm{ARL}=\frac{r}{p} \cdot \frac{1}{1-k(p)},
$$

where $\frac{r}{p}$ is the mean value of negative binomial distribution with parameters $r$ and $p$.

From an economic point of view, there is an optimal choice of parameters, which minimizes the expected total cost in control chart. In the following, an economic model will be formulated to minimize the expected total cost.

\subsection{Cost model}

Chan et al. [13], inspired by the idea of double-sampling procedures in acceptance sampling, proposed a twostage CCC-chart in order to improve one stage CCCchart. They proposed economic model to calculate the optimal values of probabilities of false alarm set at the first and second stages of two-stage CCC-chart, so that an expected total cost can be minimized.

The proposed model in this paper is based on the economic model of Chan et al. [13] in order to monitor process with low nonconforming fraction. The economic model is used to determine both chart's control limits and $r$ parameter. In addition, the sensitivity analysis is examined for cost and process parameters in two states, with and without defining constraints, which has not been addressed in Chan's model.

\subsubsection{Assumptions}

1. The process starts with a target nonconforming fraction $p_{0}$. At any moment, after the process has been started and prior to the production of the first item, or at any instant between productions of two consecutive items, one or more assignable causes will occur with probability $\pi$, which will cause the nonconforming fraction, $p$, of the process to jump from $p_{0}$ value to a larger value $p_{1}$. Consequently, the probability for this jump to occur immediately before the $i$ th produced item is:

$$
p(i)=\pi(1-\pi)^{i-1} .
$$

Such a jump, $p$, will not occur if there are no assignable causes, and will not occur during investigation of the process;

2. An item is inspected immediately after it has been produced, and the inspection always correctly reveals whether the item is conforming or nonconforming. All the nonconforming items are reworked. The cost incurred in a reworked nonconforming item is $\boldsymbol{C}_{\mathrm{rw}}$;
3. When a signal for out of control appears on the chart, then the investigation of the process will be carried out. The cost required to carry out an investigation is $C_{\text {inv }}$;

4. An investigation will be completed within a fixed period. During the period of an investigation, production will be continued, and $N$ items will be produced. During this period, if nonconforming items are produced and out-of-control signals are observed on the control chart, then there will not be additional investigations, since investigation is already carried out;

5. If no assignable cause is found at the end of an investigation, the signal for out of control that causes this investigation is a false alarm, and production will be continued;

6. When one or more assignable causes are found after an investigation is completed, then the process will be stopped and rectified. The total cost incurred in this rectification, including the cost due to the loss of production time, is $\boldsymbol{C}_{\text {rec }}$. After rectification, $p$ value will be restored to $p_{0}$ value, and production will be resumed. According to Figure 1, the production cycle assumption is as follows. A target nonconforming fraction, $p$, jumps from $p_{0}$ to $p_{1}$ after $(i-1)$ th item has been inspected immediately, including $i$ th item; $j$ items are produced altogether until a signal for out of control occurs. The small dots "..." represent inspection of items, the circle "o" denotes occurrence of a nonconforming item which does not give an out-ofcontrol signal, the heavy dot "•" denotes occurrence of a nonconforming item which gives an out-ofcontrol signal, and the star "*" indicates the start of an investigation. Figure 1 shows that during the period of investigation, $N$ items are produced.

With regard to Figure 1, production cycle can be specified now, and we can design the economic model based on production cycle;

7. Control charts allow practitioners to draw conclusions about the state of the process. These conclusions depend on whether the applied monitoring approach is in phase I or phase II of process control problem. In phase I, historical data of the process are analyzed to understand the variation of the process over time, to evaluate the process stability, and to estimate the in-control parameters.

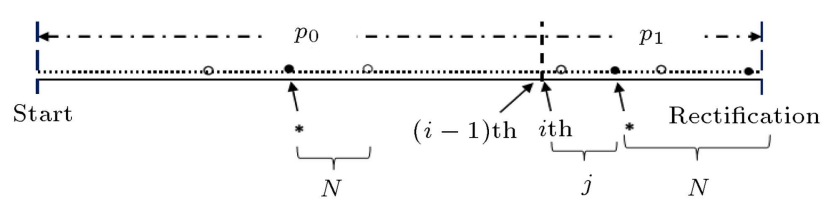

Figure 1. Shifting of the process fraction nonconforming from $p_{0}$ to $p_{1}[13]$. 
In phase II, the process is monitored in real-time to detect shifts from the baseline established in phase I quickly. The use of control charts in phase I is usually iterative. Much work, process understanding, and process improvement are often required in the transition from phase I to phase II. To measure the statistical performance of a control chart in applications of phase I, one considers the probability of any out-of-control signal in the chart to maintain a stable process. The false-alarm rate, for example, is the probability of at least one signal from the chart, given that the process is in statistical control with some assumed probability distributions. In phase II, the probability of a signal on any one sample is sometimes used if the successive statistical plotted are independent, as may be the case with a basic Shewhart-type chart. To interpret a chart in phase I, practitioners need to be aware that the probability of signals can vary considerably depending on the shape of the underlying distribution for a stable process, the degree of autocorrelation in the data, and the number of samples. Distributional and independent assumptions in theoretical studies of phase II should not be constructed as requirements in practical applications of the initial stages of phase I [23].

With respect to features of phase I and phase II discussed, the proposed model is used in phase II to monitor the process in real-time in order to detect out-of-control signals as soon as possible and consequently reduce the costs.

\subsubsection{Notations}

The following notations will be used in the formulation of the cost function for CCC- $r$ chart in this paper:

$p_{0} \quad$ In-control non-conforming fraction

$p_{1} \quad$ Out-of-control non-conforming fraction

LCL Lower Control Limit

UCL Upper Control Limit

$r \quad$ Required number of non-conforming items

$\pi \quad$ Probability for the fraction nonconforming, $p$, of the process to jump from $p_{0}$ to a larger value $p_{1}$

$N \quad$ The number of items produced during the period of investigation

$C_{\mathrm{rw}} \quad$ Cost of reworking one no-conforming item

$C_{\text {inv }} \quad$ Investigation cost
Table 1. Range of decision variables of the model.

\begin{tabular}{cc}
\hline Variable & Range \\
\hline LCL & $r+1$ to 10 \\
UCL & LCL +1 to 60 \\
$\boldsymbol{r}$ & 1 to 3 \\
\hline
\end{tabular}

$C_{\text {rec }} \quad$ Process rectification cost

$C_{\text {avg }}$ Objective function value

For decision variables, a feasible range is defined as Table 1, and they are optimized within these intervals. Cost parameters have fixed values in each experiment, as shown in Table 2, for a numerical example. By concerning these notations, the optimization model is solved to minimize the objective function by determining the optimum value for decision variables.

\subsubsection{Cost parameters}

We recall from Eq. (2) that:

$$
\begin{aligned}
& p(i)=\pi(1-\pi)^{i-1}, \\
& \sum_{i=1}^{\infty} p(i)=1, \text { and } \\
& \sum_{i=1}^{\infty}(i-1) p(i)=\frac{1-\pi}{\pi} .
\end{aligned}
$$

The different cost functions are defined as follows:

1. The cost $C_{I}$ is required in order to investigate the process when out-of-control signals are appeared. The average number of out-of-control signals that occur during the first $(i-1)$ inspected items is equal to $\frac{i-1}{\operatorname{ANI}\left(p_{0}\right)}$. Out of these signals, only a fraction $\frac{\operatorname{ANI}\left(p_{0}\right)}{\operatorname{ANI}\left(p_{0}\right)+N}$ requires investigation. Because when an out-of-control signal is observed and investigation is carried out, there will not be any additional investigation even though some other out-of-control signals are prepared before the investigation is finished.

Therefore, during the first $(i-1)$ inspected items, the average number of out-of-control signals that require investigation is $\frac{i-1}{\operatorname{ANI}\left(p_{0}\right)+N}$. After $p$ has jumped from $p_{0}$ to $p_{1}$, the $j$ th inspected item

\begin{tabular}{|c|c|c|c|c|c|c|c|c|c|c|}
\hline \multicolumn{7}{|c|}{ Process and cost parameters } & \multicolumn{3}{|c|}{ Design parameters } & \multirow{2}{*}{$\frac{\text { Objective function }}{C_{\text {avg }}}$} \\
\hline$p_{0}$ & $p_{1}$ & $C_{\text {inv }}$ & $C_{\mathrm{rw}}$ & $C_{\text {rec }}$ & $\pi$ & $N$ & LCL & UCL & $\boldsymbol{r}$ & \\
\hline 0.1 & 0.125 & 0.5 & 50 & 400 & 0.2 & 10 & 4 & 60 & 3 & 7.252376 \\
\hline
\end{tabular}
shows an out-of-control signal which requires an

Table 2. Optimal control chart design and cost parameters according to a specified $p_{0}$ and $p_{1}$. 
investigation with cost $C_{\text {inv }}$. Therefore:

$$
C_{I}=\frac{C_{\mathrm{inv}}(i-1)}{\left(\operatorname{ANI}\left(p_{0}\right)+N\right)}+C_{\mathrm{inv}}
$$

and:

$$
\begin{aligned}
E\left(C_{I}\right) & =C_{\mathrm{inv}}\left(\sum_{i=1}^{\infty} \frac{(i-1) p(i)}{\left(\operatorname{ANI}\left(p_{0}\right)+N\right)}\right)+C_{\mathrm{inv}} \\
& =C_{\mathrm{inv}} \frac{1-\pi}{\left(\pi\left(\operatorname{ANI}\left(p_{0}\right)+N\right)\right)}+C_{\mathrm{inv}} .
\end{aligned}
$$

2. The cost $C_{R}$ is required to rework all the nonconforming items found in inspection: During the first $(i-1)$ inspected items, the nonconforming fraction of the process is $p_{0}$. Therefore, out of these $(i-1)$ inspected items, the average number of nonconforming items is $(i-1) p_{0}$, and these nonconforming items require rework. Such a rework requires a cost:

$$
C_{R 1}=C_{\mathrm{rw}} \sum_{i=1}^{\infty}(i-1) p(i) p_{0}=C_{\mathrm{rw}} \frac{1-\pi}{\pi} p_{0} .
$$

The total number of produced items after $(i-1)$ th inspected item and until the complete investigation is $(j+N)$, and the expected number of nonconforming produced items is $(j+N) p_{1}$. The expected cost required to rework all these items is:

$$
C_{R 2}=C_{\mathrm{rw}} \sum_{j=1}^{\infty}(j+N) p_{1} k(p)=C_{\mathrm{rw}}\left(\operatorname{ANI}\left(p_{1}\right)+N\right),
$$

where $\operatorname{ANI}\left(p_{1}\right)$ is the expected number of items produced before observing an alarm, and $N$ is the expected number of items produced during investigation .

Thus, the expected total cost of rework is:

$$
\begin{aligned}
E\left(C_{R}\right)= & C_{R 1}+C_{R 2}=C_{\mathrm{rw}}\left(\frac{1-\pi}{\pi} p_{0}\right. \\
& \left.+p_{1}\left(\operatorname{ANI}\left(p_{1}\right)+N\right)\right)
\end{aligned}
$$

3. The cost $C_{\text {rec }}$ is required to rectify the process when one or more assignable causes occur.

\subsubsection{Objective function}

The expected total cost in cycle is $E\left(C_{I}\right)+E\left(C_{R}\right)+$ $C_{\text {rec }}$. The total number of produced items in a production cycle is $(i-1)+j+N$, and its expected value is:

$$
\begin{aligned}
E((i-1)+j+N) & =E(i-1)+\operatorname{ANI}\left(p_{1}\right)+N \\
& =\sum_{i=1}^{\infty}(i-1) p(i)+\operatorname{ANI}\left(p_{1}\right)+N \\
& =\frac{1-\pi}{\pi}+\operatorname{ANI}\left(p_{1}\right)+N .
\end{aligned}
$$

At last, the objective cost function is the average cost per produced item for two-sided CCC- $r$ control chart. This function is based on the expected cycle length and cost:

$$
\begin{aligned}
C_{\mathrm{avg}}= & \frac{C_{\mathrm{inv}}\left(1+\frac{1-\pi}{\pi}\left(\frac{1}{\operatorname{ANI}\left(p_{0}\right)+N}\right)\right)}{\frac{1-\pi}{\pi}+\operatorname{ANI}\left(p_{1}\right)+N} \\
& +\frac{C_{\mathrm{rw}}\left(\frac{1-\pi}{\pi}\left(p_{0}\right)+p_{1}\left(\operatorname{ANI}\left(p_{0}\right)+N\right)\right)}{\frac{1-\pi}{\pi}+\operatorname{ANI}\left(p_{1}\right)+N} \\
& +\frac{C_{\mathrm{rec}}}{\frac{1-\pi}{\pi}+\operatorname{ANI}\left(p_{1}\right)+N} .
\end{aligned}
$$

All the parameters are constant and by defining a custom range for decision variable in the model, the optimal values are determined, so that all related costs per one item in production cycle could be minimized. To better illustrate and examine the model, we will solve a numerical example in the next section.

\section{Application of the model in an example}

With respect to the definition and assumptions in the cost model, we used visual basic to design a search procedure to find the optimum value of control limits and $\mathrm{r}$ as decision variables according to their specified range in Table 1 . This method begins with the first possible value of $r$, then LCL, and finally UCL values are selected in the specified intervals. These values are placed in objective. The UCL value is changed until all possible values in its range are examined while LCL and $r$ values are fixed. After considering all UCL values, next possible value for LCL with all possible values of UCL is examined while $r$ is fixed. When all possible combinations of UCL and LCL are checked, $r$ is changed. By this method, all different combinations of decision variables, which are 2245 possible cases, will be investigated. Finally, the minimum value of objective function among these cases will be the optimum value.

According to the cost and process parameters in the specified control chart, as is shown in Table 2, the minimum of average cost per produced item is 7.047593396. This optimum value is achieved when $r$ is equal to 3 , lower control limit is equal to 4 , and upper control limit is equal to 60 . If the number of inspected items is less than 4 , it is indicative of a problem in process, so that the nonconforming proportion is increased, and it is needed to examine the process for detecting assignable causes. Also, if the number of inspected items is more than 60, it indicates improvement in process quality.

Based on the result in Table 2, it is clear that the optimal solutions are not practically suitable. The 
process will be stopped and examined when 3 nonconforming items are found among 4 inspected items. This result contradicts the assumption of high-quality processes. The reason for such problems is the ignoring of statistical features and risk constraints in the economic model. In the following sections, risk constraints will be added to the proposed model, and it attempts to make the model closer to the assumption of highquality processes.

In the next section, we survey optimal design parameters for different values of $p_{0}$ and $p_{1}$, cost, and process parameters as denoted in Table 2, which will change separately in each iteration.

\subsection{Model sensitivity analysis}

3.1.1. Sensitivity analysis of design parameters based on different $p_{0}$ and $p_{1}$

Table 3 provides the optimal solutions and minimum average cost per produced item in different scenarios of parameters. The optimal solutions and objective function have been calculated in each scenario.

Thus, Table 3 can be used to analyze the sensitivity of the model to the various parameters. With regard to the results, $N$ and $\pi$ have a reverse result on objective value, so that in experiments where the differences between $p_{0}$ and $p_{1}$ are low, the variation of objective function with respect to the variations of these parameters is little, but when the difference between them becomes larger, decreasing the parameters $N$ and $\pi$ leads to the further increase of objective function. In addition, $N$ impact is much more.

Variations of objective function are directly related to all variation of cost parameters in the model. According to Table $3, C_{\text {rec }}$ and $C_{\text {rw }}$ have more impact on the objective function, but the relation $C_{\text {inv }}$ does not significantly change it. Effect of $C_{\text {rw }}$ and $C_{\text {rec }}$ on objective function is greater. As shown in Table 3, increasing cost parameters leads larger lower control limit in each experiment. Furthermore, with a fixed value of $p_{0}$, the upper control limit decreases as the value of $p_{1}$ is increased.

\subsubsection{Sensitivity analysis of design parameters based on $A N I$ in the process}

In this section, with regard to the acceptable false alarm probability (Type I error) and observing process as in control while the process is actually out of control (Type II error), optimal design parameters are determined by defining the constraints for ANI in the cases that process is in control and out of control. With regard to the definitions of $\alpha$ and $\beta$ in statistical process control and equations related to process which have been defined in the previous sections, two constraints on ANI based on $\alpha$ and $\beta$ would be added to the model in each experiment. The constraints are defined as follows:

$$
\begin{aligned}
& k\left(p_{0}\right)=\sum_{i=\mathrm{LCL}}^{\mathrm{UCL}}\left(\begin{array}{l}
i-1 \\
r-1
\end{array}\right) p_{0}^{r}\left(1-p_{0}\right)^{i-r} \geq 1-\alpha, \\
& k\left(p_{1}\right)=\sum_{i=\mathrm{LCL}}^{\mathrm{UCL}}\left(\begin{array}{l}
i-1 \\
r-1
\end{array}\right) p_{1}^{r}\left(1-p_{1}\right)^{i-r} \leq \beta .
\end{aligned}
$$

Eqs. (3) and (4) can be revised according to formulations and properties of CCC- $r$ control chart, and they can be rewritten as the constraints $\operatorname{ANI}\left(p_{0}\right) \geq \frac{1}{\alpha}$ and $\operatorname{ANI}\left(p_{1}\right) \leq \frac{1}{1-\beta}$. Now, the feasible search space of the model becomes limited.

As shown in Table 4, in Experiments 1 to 5, design parameters are changed clearly. Despite larger objective value in comparison with the results in Table 3 , the process identifies nonconforming items LCL, UCL, and $\mathrm{r}$ values, which are economically and statistically optimal and can be practically more useful.

\section{Discussions}

\subsection{Discussion 1: Inspection errors}

With regard to Xie et al. [22], an inspection operation may result in two types of inspection errors: classification of a conforming item as nonconforming and classification of a nonconforming item as conforming. Because of the errors involved in the inspection of items, process nonconforming fraction might deviate from its true value. In this condition, we have relationship between the true and observed process fraction nonconforming as follows:

$$
p_{t}=\frac{p_{o}-\theta}{1-\theta-\varphi} .
$$

And accordingly:

$$
p_{o}=p_{t}-p_{t} \varphi+\left(1-p_{t}\right) \theta,
$$

where $p_{o}$ and $p_{t}$ represent the observed $p$ value and true value, respectively, while $\theta$ and $\varphi$, respectively, denote the probability of classifying a conforming item as nonconforming and the probability of classifying a nonconforming item as conforming. It is clear that for a fixed $\varphi$ value, $p_{t}$ approaches zero when inspection error, $\theta$, approaches $p_{o}$. The inspection error $\theta$ tends to increase $p_{o}$, while inspection error $\varphi$ tends to reduce $p_{o}$. In addition, the inspection error $\theta$ affects $p_{o}$ more significantly in comparison to what the inspection error $\varphi$ does, as the value of $\left(1-p_{t}\right)$ is commonly larger than that of $p_{t}$.

By considering inspection errors, false alarm probability and subsequently average run length will be changed:

$$
\alpha_{\text {actual }}=1-p(\mathrm{LCL} \leq x \leq \mathrm{UCL}),
$$

where this equation can be calculated with respect to $p_{o}$ equation. In-control ARL can be calculated as below: 
Table 3. Optimal control chart design and cost parameters according to different $p_{0}$ and $p_{1}$.

\begin{tabular}{|c|c|c|c|c|c|c|c|c|c|c|c|c|}
\hline \multirow{2}{*}{\multicolumn{2}{|c|}{ Exp. no. }} & \multicolumn{7}{|c|}{ Process and cost parameters } & \multicolumn{3}{|c|}{ Design parameters } & \multirow{2}{*}{$\frac{\text { Objective function }}{C_{\mathrm{avg}}}$} \\
\hline & & $p_{0}$ & $p_{1}$ & $C_{\text {inv }}$ & $C_{\mathrm{rw}}$ & $C_{\text {rec }}$ & $\pi$ & $N$ & LCL & UCL & $\boldsymbol{r}$ & \\
\hline \multirow[t]{3}{*}{1} & \multirow{3}{*}{$\pi$} & 0.05 & 0.125 & 0.5 & 50 & 400 & 0.2 & 10 & 4 & 60 & 3 & 7.228529972 \\
\hline & & 0.05 & 0.125 & 0.5 & 50 & 400 & 0.1 & 10 & 4 & 60 & 3 & 7.258833811 \\
\hline & & 0.05 & 0.125 & 0.5 & 50 & 400 & 0.3 & 10 & 4 & 60 & 3 & 7.218593924 \\
\hline & \multirow{2}{*}{$N$} & 0.05 & 0.125 & 0.5 & 50 & 400 & 0.2 & 5 & 4 & 60 & 3 & 7.270501205 \\
\hline & & 0.05 & 0.125 & 0.5 & 50 & 400 & 0.2 & 20 & 4 & 60 & 3 & 7.234861486 \\
\hline & \multirow{2}{*}{$C_{\mathrm{inv}}$} & 0.05 & 0.125 & 0.25 & 50 & 400 & 0.2 & 10 & 4 & 60 & 3 & 7.227934039 \\
\hline & & 0.05 & 0.125 & 1 & 50 & 400 & 0.2 & 10 & 4 & 60 & 3 & 7.229721836 \\
\hline & \multirow{2}{*}{$C_{\mathrm{rw}}$} & 0.05 & 0.125 & 0.5 & 25 & 400 & 0.2 & 10 & 4 & 60 & 3 & 4.091611327 \\
\hline & & 0.05 & 0.125 & 0.5 & 100 & 400 & 0.2 & 10 & 4 & 60 & 3 & 13.50236726 \\
\hline & \multirow{2}{*}{$C_{\text {rec }}$} & 0.05 & 0.125 & 0.5 & 100 & 200 & 0.2 & 10 & 4 & 60 & 3 & 6.751784175 \\
\hline & & 0.05 & 0.125 & 0.5 & 100 & 800 & 0.2 & 10 & 4 & 60 & 3 & 8.182021565 \\
\hline \multirow[t]{11}{*}{2} & \multirow{3}{*}{$\pi$} & 0.05 & 0.2 & 0.5 & 50 & 400 & 0.2 & 10 & 4 & 60 & 3 & 13.6933591 \\
\hline & & 0.05 & 0.2 & 0.5 & 50 & 400 & 0.1 & 10 & 4 & 60 & 3 & 13.8028148 \\
\hline & & 0.05 & 0.2 & 0.5 & 50 & 400 & 0.3 & 10 & 4 & 60 & 3 & 13.65284295 \\
\hline & \multirow{2}{*}{$N$} & 0.05 & 0.2 & 0.5 & 50 & 400 & 0.2 & 5 & 4 & 60 & 3 & 13.86401771 \\
\hline & & 0.05 & 0.2 & 0.5 & 50 & 400 & 0.2 & 20 & 4 & 60 & 3 & 13.38594907 \\
\hline & \multirow{2}{*}{$C_{\text {inv }}$} & 0.05 & 0.2 & 0.25 & 50 & 400 & 0.2 & 10 & 4 & 60 & 3 & 13.6880885 \\
\hline & & 0.05 & 0.2 & 1 & 50 & 400 & 0.2 & 10 & 4 & 60 & 3 & 13.6948374 \\
\hline & & 0.05 & 0.2 & 0.5 & 25 & 400 & 0.2 & 10 & 4 & 60 & 3 & 8.64538789 \\
\hline & $C_{\mathrm{rw}}$ & 0.05 & 0.2 & 0.5 & 100 & 400 & 0.2 & 10 & 4 & 60 & 3 & 23.78 .23236 \\
\hline & & 0.05 & 0.2 & 0.5 & 50 & 200 & 0.2 & 10 & 4 & 60 & 3 & 11.8924 .690 \\
\hline & $C_{\mathrm{rec}}$ & 0.05 & 0.2 & 0.5 & 50 & 800 & 0.2 & 10 & 4 & 60 & 3 & 17.2861939 \\
\hline 3 & & 0.1 & 0.125 & 0.5 & 50 & 400 & 0.2 & 10 & 4 & 60 & 3 & 7.252376308 \\
\hline & $\pi$ & 0.1 & 0.125 & 0.5 & 50 & 400 & 0.1 & 10 & 4 & 60 & 3 & 7.31199237 \\
\hline & & 0.1 & 0.125 & 0.5 & 50 & 400 & 0.3 & 10 & 4 & 60 & 3 & 7.232504287 \\
\hline & $N$ & 0.1 & 0.125 & 0.5 & 50 & 400 & 0.2 & 5 & 4 & 60 & 3 & 7.26447563 \\
\hline & 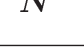 & 0.1 & 0.125 & 0.5 & 50 & 400 & 0.2 & 20 & 4 & 60 & 3 & 7.229038344 \\
\hline & & 0.1 & 0.125 & 0.25 & 50 & 400 & 0.2 & 10 & 4 & 60 & 3 & 7.251780376 \\
\hline & $C$ inv & 0.1 & 0.125 & 1 & 50 & 400 & 0.2 & 10 & 4 & 60 & 3 & 7.253568173 \\
\hline & & 0.1 & 0.125 & 0.5 & 25 & 400 & 0.2 & 10 & 4 & 60 & 3 & 4.103539018 \\
\hline & $C_{\mathrm{rw}}$ & 0.1 & 0.125 & 0.5 & 100 & 400 & 0.2 & 10 & 4 & 60 & 3 & 13.555089 \\
\hline & $C$ & 0.1 & 0.125 & 0.5 & 50 & 200 & 0.2 & 10 & 4 & 60 & 3 & 6.775630511 \\
\hline & Crec & 0.1 & 0.125 & 0.5 & 50 & 800 & 0.2 & 10 & 4 & 60 & 3 & 8.205867902 \\
\hline 4 & & 0.1 & 0.2 & 0.5 & 50 & 400 & 0.2 & 10 & 4 & 60 & 3 & 13.7831494 \\
\hline & $\pi$ & 0.1 & 0.2 & 0.5 & 50 & 400 & 0.1 & 10 & 4 & 60 & 3 & 14.00526761 \\
\hline & & 0.1 & 0.2 & 0.5 & 50 & 400 & 0.3 & 10 & 4 & 60 & 3 & 13.70533072 \\
\hline & $N$ & 0.1 & 0.2 & 0.5 & 50 & 400 & 0.2 & 5 & 4 & 60 & 3 & 13.9582364 \\
\hline & N & 0.1 & 0.2 & 0.5 & 50 & 400 & 0.2 & 20 & 4 & 60 & 3 & 13.64850138 \\
\hline & $C$ & 0.1 & 0.2 & 0.25 & 50 & 400 & 0.2 & 10 & 4 & 60 & 3 & 13.7706753 \\
\hline & Cinv & 0.1 & 0.2 & 1 & 50 & 400 & 0.2 & 10 & 4 & 60 & 3 & 13.78480976 \\
\hline & $C$ & 0.1 & 0.2 & 0.5 & 25 & 400 & 0.2 & 10 & 4 & 60 & 3 & 8.69 .418492 \\
\hline & $C_{\mathrm{rw}}$ & 0.1 & 0.2 & 0.5 & 100 & 400 & 0.2 & 10 & 4 & 60 & 3 & 23.96 .10784 \\
\hline & & 0.1 & 0.2 & 0.5 & 50 & 200 & 0.2 & 10 & 4 & 60 & 3 & 11.98238595 \\
\hline & $C_{\text {rec }}$ & 0.1 & 0.2 & 0.5 & 50 & 800 & 0.2 & 10 & 4 & 60 & 3 & 17.37617293 \\
\hline 5 & & 0.1 & 0.3 & 0.5 & 50 & 400 & 0.2 & 10 & 4 & 60 & 3 & 25.97042044 \\
\hline & $\pi$ & 0.1 & 0.3 & 0.5 & 50 & 400 & 0.1 & 10 & 4 & 60 & 3 & 26.62395283 \\
\hline & & 0.1 & 0.3 & 0.5 & 50 & 400 & 0.3 & 10 & 4 & 60 & 3 & 25.75257631 \\
\hline & $N$ & 0.1 & 0.3 & 0.5 & 50 & 400 & 0.2 & 5 & 4 & 60 & 3 & 27.61601173 \\
\hline & $N$ & 0.1 & 0.3 & 0.5 & 50 & 400 & 0.2 & 20 & 4 & 60 & 3 & 23.70063522 \\
\hline & $C_{\text {inn }}$ & 0.1 & 0.3 & 0.25 & 50 & 400 & 0.2 & 10 & 4 & 60 & 3 & 25.96389884 \\
\hline & $C_{\text {inv }}$ & 0.1 & 0.3 & 1 & 50 & 400 & 0.2 & 10 & 4 & 60 & 3 & 25.98346362 \\
\hline & $C$ & 0.1 & 0.3 & 0.5 & 25 & 400 & 0.2 & 10 & 4 & 60 & 3 & 18.20955666 \\
\hline & $C_{r w}$ & 0.1 & 0.3 & 0.5 & 100 & 400 & 0.2 & 10 & 4 & 60 & 3 & 41.49214801 \\
\hline & & 0.1 & 0.3 & 0.5 & 50 & 200 & 0.2 & 10 & 4 & 60 & 3 & 20.75314477 \\
\hline & $C_{\text {rec }}$ & 0.1 & 0.3 & 0.5 & 50 & 800 & 0.2 & 10 & 4 & 60 & 3 & 36.40497178 \\
\hline
\end{tabular}


Table 3. Optimal control chart design and cost parameters according to different $p_{0}$ and $p_{1}$ (continued).

\begin{tabular}{|c|c|c|c|c|c|c|c|c|c|c|c|c|}
\hline \multirow{2}{*}{\multicolumn{2}{|c|}{ Exp. no. }} & \multicolumn{7}{|c|}{ Process and cost parameters } & \multicolumn{3}{|c|}{ Design parameters } & \multirow{2}{*}{$\frac{\text { Objective function }}{C_{\text {avg }}}$} \\
\hline & & $p_{0}$ & $p_{1}$ & $C_{\text {inv }}$ & $C_{\mathrm{rw}}$ & $C_{\text {rec }}$ & $\pi$ & $N$ & LCL & UCL & $\boldsymbol{r}$ & \\
\hline \multirow[t]{11}{*}{6} & \multirow{3}{*}{$\pi$} & 0.1 & 0.4 & 0.5 & 50 & 400 & 0.2 & 10 & 4 & 54 & 3 & 38.34213215 \\
\hline & & 0.1 & 0.4 & 0.5 & 50 & 400 & 0.1 & 10 & 4 & 54 & 3 & 39.43599286 \\
\hline & & 0.1 & 0.4 & 0.5 & 50 & 400 & 0.3 & 10 & 4 & 56 & 3 & 37.97751191 \\
\hline & \multirow{2}{*}{$N$} & 0.1 & 0.4 & 0.5 & 50 & 400 & 0.2 & 5 & 4 & 56 & 3 & 43.45769432 \\
\hline & & 0.1 & 0.4 & 0.5 & 50 & 400 & 0.2 & 20 & 4 & 56 & 3 & 32.7716336 \\
\hline & \multirow{2}{*}{$C_{\text {inv }}$} & 0.1 & 0.4 & 0.25 & 50 & 400 & 0.2 & 10 & 4 & 53 & 3 & 38.33122888 \\
\hline & & 0.1 & 0.4 & 1 & 50 & 400 & 0.2 & 10 & 4 & 53 & 3 & 38.3639387 \\
\hline & \multirow{2}{*}{$C_{\mathrm{rw}}$} & 0.1 & 0.4 & 0.5 & 25 & 400 & 0.2 & 10 & 4 & 55 & 3 & 45.7836918 \\
\hline & & 0.1 & 0.4 & 0.5 & 100 & 400 & 0.2 & 10 & 4 & 55 & 3 & 59.214394 \\
\hline & \multirow{2}{*}{$C_{\text {rec }}$} & 0.1 & 0.4 & 0.5 & 50 & 200 & 0.2 & 10 & 4 & 54 & 3 & 29.61951363 \\
\hline & & 0.1 & 0.4 & 0.5 & 50 & 800 & 0.2 & 10 & 4 & 60 & 3 & 55.7836918 \\
\hline \multirow[t]{11}{*}{7} & \multirow{3}{*}{$\pi$} & 0.1 & 0.5 & 0.5 & 50 & 400 & 0.2 & 10 & 4 & 39 & 3 & 48.68465118 \\
\hline & & 0.1 & 0.5 & 0.5 & 50 & 400 & 0.1 & 10 & 4 & 39 & 3 & 50.09827232 \\
\hline & & 0.1 & 0.5 & 0.5 & 50 & 400 & 0.3 & 10 & 4 & 40 & 3 & 48.21344412 \\
\hline & \multirow{2}{*}{$N$} & 0.1 & 0.5 & 0.5 & 50 & 400 & 0.2 & 5 & 4 & 41 & 3 & 57.96796801 \\
\hline & & 0.1 & 0.5 & 0.5 & 50 & 400 & 0.2 & 20 & 4 & 42 & 3 & 40.15146887 \\
\hline & \multirow{2}{*}{$C_{\text {inv }}$} & 0.1 & 0.5 & 0.25 & 50 & 400 & 0.2 & 10 & 4 & 39 & 3 & 48.67057268 \\
\hline & & 0.1 & 0.5 & 1 & 50 & 400 & 0.2 & 10 & 4 & 41 & 3 & 48.71280817 \\
\hline & $C$ & 0.1 & 0.5 & 0.5 & 25 & 400 & 0.2 & 10 & 4 & 39 & 3 & 35.6215125 \\
\hline & $C_{r w}$ & 0.1 & 0.5 & 0.5 & 100 & 400 & 0.2 & 10 & 4 & 40 & 3 & 74.81093104 \\
\hline & & 0.1 & 0.5 & 0.5 & 50 & 200 & 0.2 & 10 & 4 & 40 & 3 & 37.42185284 \\
\hline & $C_{\text {rec }}$ & 0.1 & 0.5 & 0.5 & 50 & 800 & 0.2 & 10 & 4 & 40 & 3 & 71.21024845 \\
\hline 8 & & 0.2 & 0.3 & 0.5 & 50 & 400 & 0.2 & 10 & 4 & 60 & 3 & 26.49303715 \\
\hline & $\pi$ & 0.2 & 0.3 & 0.5 & 50 & 400 & 0.1 & 10 & 4 & 60 & 3 & 27.79984042 \\
\hline & & 0.2 & 0.3 & 0.5 & 50 & 400 & 0.3 & 10 & 4 & 60 & 3 & 26.05743606 \\
\hline & $N$ & 0.2 & 0.3 & 0.5 & 50 & 400 & 0.2 & 5 & 4 & 60 & 3 & 28.21718936 \\
\hline & & 0.2 & 0.3 & 0.5 & 50 & 400 & 0.2 & 20 & 4 & 60 & 3 & 24.11495699 \\
\hline & & 0.2 & 0.3 & 0.25 & 50 & 400 & 0.2 & 10 & 4 & 60 & 3 & 26.48651555 \\
\hline & $C_{\text {inv }}$ & 0.2 & 0.3 & 1 & 50 & 400 & 0.2 & 10 & 4 & 60 & 3 & 26.50608003 \\
\hline & & 0.2 & 0.3 & 0.5 & 25 & 400 & 0.2 & 10 & 4 & 60 & 3 & 18.47130958 \\
\hline & $C_{r w}$ & 0.2 & 0.3 & 0.5 & 100 & 400 & 0.2 & 10 & 4 & 60 & 3 & 42.53649228 \\
\hline & $C$ & 0.2 & 0.3 & 0.5 & 50 & 200 & 0.2 & 10 & 4 & 60 & 3 & 21.27576148 \\
\hline & $C_{\text {rec }}$ & 0.2 & 0.3 & 0.5 & 50 & 800 & 0.2 & 10 & 4 & 60 & 3 & 36.92758848 \\
\hline 9 & & 0.2 & 0.4 & 0.5 & 50 & 400 & 0.2 & 10 & 4 & 57 & 3 & 39.2164324 \\
\hline & $\pi$ & 0.2 & 0.4 & 0.5 & 50 & 400 & 0.1 & 10 & 4 & 55 & 3 & 41.4036843 \\
\hline & & 0.2 & 0.4 & 0.5 & 50 & 400 & 0.3 & 10 & 4 & 59 & 3 & 38.48752039 \\
\hline & $N$ & 0.2 & 0.4 & 0.5 & 50 & 400 & 0.2 & 5 & 4 & 55 & 3 & 44.5765084 \\
\hline & $N$ & 0.2 & 0.4 & 0.5 & 50 & 400 & 0.2 & 20 & 4 & 55 & 3 & 33.37995801 \\
\hline & $C$ & 0.2 & 0.4 & 0.25 & 50 & 400 & 0.2 & 10 & 4 & 55 & 3 & 26.12160135 \\
\hline & Cinv & 0.2 & 0.4 & 1 & 50 & 400 & 0.2 & 10 & 4 & 54 & 3 & 26.15431117 \\
\hline & $C$ & 0.2 & 0.4 & 0.5 & 25 & 400 & 0.2 & 10 & 4 & 52 & 3 & 28.34417055 \\
\hline & $C_{r w}$ & 0.2 & 0.4 & 0.5 & 100 & 400 & 0.2 & 10 & 4 & 55 & 3 & 60.96095611 \\
\hline & & 0.2 & 0.4 & 0.5 & 50 & 200 & 0.2 & 10 & 4 & 55 & 3 & 30.49381389 \\
\hline & $C_{\text {rec }}$ & 0.2 & 0.4 & 0.5 & 50 & 800 & 0.2 & 10 & 4 & 54 & 3 & 56.66166944 \\
\hline 10 & & 0.2 & 0.5 & 0.5 & 50 & 400 & 0.2 & 10 & 4 & 40 & 3 & 49.79989884 \\
\hline & $\pi$ & 0.2 & 0.5 & 0.5 & 50 & 400 & 0.1 & 10 & 4 & 42 & 3 & 52.6325618 \\
\hline & & 0.2 & 0.5 & 0.5 & 50 & 400 & 0.3 & 10 & 4 & 39 & 3 & 48.87221177 \\
\hline & $N$ & 0.2 & 0.5 & 0.5 & 50 & 400 & 0.2 & 5 & 4 & 42 & 3 & 59.54135672 \\
\hline & $N$ & 0.2 & 0.5 & 0.5 & 50 & 400 & 0.2 & 20 & 4 & 38 & 3 & 40.87319953 \\
\hline & $C_{\mathrm{inn}}$ & 0.2 & 0.5 & 0.25 & 50 & 400 & 0.2 & 10 & 4 & 41 & 3 & 49.79989884 \\
\hline & Cinv & 0.2 & 0.5 & 1 & 50 & 400 & 0.2 & 10 & 4 & 39 & 3 & 49.84213434 \\
\hline & $C$ & 0.2 & 0.5 & 0.5 & 25 & 400 & 0.2 & 10 & 4 & 39 & 3 & 36.18769748 \\
\hline & $C_{r w}$ & 0.2 & 0.5 & 0.5 & 100 & 400 & 0.2 & 10 & 4 & 39 & 3 & 77.06653707 \\
\hline & & 0.2 & 0.5 & 0.5 & 50 & 200 & 0.2 & 10 & 4 & 38 & 3 & 38.55117871 \\
\hline & $C_{\text {rec }}$ & 0.2 & 0.5 & 0.5 & 50 & 800 & 0.2 & 10 & 4 & 41 & 3 & 72.33957461 \\
\hline
\end{tabular}


Table 4. Optimal control chart design and cost parameters according to Type I error and Type II error.

\begin{tabular}{|c|c|c|c|c|c|c|c|c|c|c|c|c|c|}
\hline \multirow{3}{*}{$\begin{array}{c}\text { Exp. } \\
\text { no. } \\
1 \\
1\end{array}$} & \multicolumn{9}{|c|}{ Process and cost parameters } & \multicolumn{3}{|c|}{ Design parameters } & \multirow{2}{*}{$\begin{array}{c}\begin{array}{c}\text { Objective } \\
\text { function }\end{array} \\
C_{\mathrm{avg}} \\
\end{array}$} \\
\hline & $p_{0}$ & $p_{1}$ & $C_{\text {inv }}$ & $C_{\text {rw }}$ & $C_{\text {rec }}$ & $\pi$ & $N$ & $\operatorname{ANI}\left(p_{0}\right)>=150$ & $\operatorname{ANI}\left(p_{1}\right)<=70$ & LCL & UCL & $r$ & \\
\hline & 0.05 & 0.125 & 50 & 0.5 & 400 & 0.2 & 10 & 174.6462262 & 69.858495 & 4 & 33 & 2 & 11.39061139 \\
\hline \multirow[b]{2}{*}{2} & $p_{0}$ & $p_{1}$ & $C_{\text {inv }}$ & $C_{\mathrm{rw}}$ & $C_{\mathrm{rec}}$ & $\pi$ & $N$ & $\operatorname{ANI}\left(p_{0}\right)>=150$ & $\operatorname{ANI}\left(p_{1}\right)<=40$ & LCL & UCL & $r$ & \\
\hline & 0.05 & 0.2 & 50 & 0.5 & 400 & 0.2 & 10 & 159.438825 & 39.8597062 & 6 & 22 & 3 & 18.23357453 \\
\hline \multirow[b]{2}{*}{3} & $p_{0}$ & $p_{1}$ & $C_{\text {inv }}$ & $C_{\mathrm{rw}}$ & $C_{\mathrm{rec}}$ & $\pi$ & $N$ & $\operatorname{ANI}\left(p_{0}\right)>=240$ & $\operatorname{ANI}\left(p_{1}\right)<=200$ & $\mathrm{LCL}$ & UCL & $r$ & $C_{\text {avg }}$ \\
\hline & 0.1 & 0.125 & 50 & 0.5 & 400 & 0.2 & 10 & 247.9903027 & 198.392242 & 4 & 28 & 3 & 8.2679 \\
\hline \multirow[b]{2}{*}{4} & $p_{0}$ & $p_{1}$ & $C_{\mathrm{inv}}$ & $C_{\mathrm{rw}}$ & $C_{\text {rec }}$ & $\pi$ & $N$ & $\operatorname{ANI}\left(p_{0}\right)>80$ & $\operatorname{ANI}\left(p_{1}\right)<=41$ & LCL & UCL & $r$ & $C_{\mathrm{a}}$ \\
\hline & 0.1 & 0.2 & 50 & 0.5 & 400 & 0.2 & 10 & & 40.9411569 & 7 & 28 & 3 & 18.25547622 \\
\hline \multirow[b]{2}{*}{5} & $p_{0}$ & $p_{1}$ & $C_{\text {inv }}$ & $C_{\mathrm{rw}}$ & $C_{\text {rec }}$ & $\pi$ & $N$ & $\operatorname{ANI}\left(p_{0}\right)>=35$ & $\operatorname{ANI}\left(p_{1}\right)<=8$ & LCL & UCL & $r$ & $C_{\text {avg }}$ \\
\hline & 0.1 & 0.3 & 50 & 0.5 & 400 & 0.2 & 10 & 38.78787877 & 7.75757575 & 4 & 39 & 2 & 48.68465118 \\
\hline \multirow[b]{2}{*}{6} & $p_{0}$ & $p_{1}$ & $C_{\text {inv }}$ & $C_{\mathrm{rw}}$ & $C_{\mathrm{rec}}$ & $\pi$ & $N$ & $\operatorname{ANI}\left(p_{0}\right)>=50$ & $\operatorname{ANI}\left(p_{1}\right)<=13$ & LCL & UCL & $r$ & $C_{\text {avg }}$ \\
\hline & 0.1 & 0.4 & 50 & 0.5 & 400 & 0.2 & 10 & 51.71557809 & 12.928845 & 4 & 54 & 3 & 38.342 \\
\hline & $p_{0}$ & $p_{1}$ & $C_{\text {inv }}$ & $C_{\mathrm{rw}}$ & $C_{\text {rec }}$ & $\pi$ & $N$ & $\operatorname{ANI}\left(p_{0}\right)>=30$ & $\operatorname{ANI}\left(p_{1}\right)<=7$ & LCL & UCL & $r$ & $C_{\text {avg }}$ \\
\hline & 0.1 & 0.5 & 50 & 0.5 & 400 & 0.2 & 10 & 34.09830074 & 6.9966148 & 5 & 14 & 3 & 49.74 \\
\hline \multirow[b]{2}{*}{8} & $p_{0}$ & $\mathrm{p}_{1}$ & $C_{\text {inv }}$ & $C_{\mathrm{rw}}$ & $C_{\text {rec }}$ & $\pi$ & $N$ & $\operatorname{ANI}\left(p_{0}\right)>=27$ & $\operatorname{ANI}\left(p_{1}\right)<=19$ & LCL & UCL & $r$ & \\
\hline & 0.2 & 0.3 & 50 & 0.5 & 400 & 0.2 & 10 & 27.92417662 & 18.6161177 & 6 & 60 & 3 & 30.3910863 \\
\hline & $p_{0}$ & $p_{1}$ & $C_{\text {inv }}$ & $C_{\mathrm{rw}}$ & $C_{\text {rec }}$ & $\pi$ & $N$ & $\operatorname{ANI}\left(p_{0}\right)>=21$ & $\operatorname{ANI}\left(p_{1}\right)<=11$ & LCL & UCL & $r$ & \\
\hline & 0.2 & 0.4 & 50 & 0.5 & 400 & 0.2 & 10 & 21.09104306 & 10.9552153 & 5 & 53 & 3 & 41.02700184 \\
\hline \multirow[b]{2}{*}{10} & $p_{0}$ & $p_{1}$ & $C_{\text {inv }}$ & $C_{\mathrm{rw}}$ & $C_{\mathrm{rec}}$ & $\pi$ & $N$ & $\operatorname{ANI}\left(p_{0}\right)>=19$ & $\operatorname{ANI}\left(p_{1}\right)<=8$ & LCL & UCL & $r$ & \\
\hline & 0.2 & 0.5 & 50 & 0.5 & 400 & 0.2 & 10 & 19.39393939 & 7.75757576 & 4 & 40 & 3 & 49.81397734 \\
\hline
\end{tabular}

$$
\mathrm{ARL}=\frac{1}{\alpha_{\text {actual }}}=\frac{1}{1-k\left(p_{o}\right)}
$$

According to ARL, ANI, which is performance index in the proposed economic model, can be written as:

$$
\operatorname{ANI}\left(p_{t}, \theta, \varphi\right)=\frac{r}{p_{o}} * \frac{1}{1-k\left(p_{o}\right)} .
$$

Thus, to modify the economic model to consider inspection errors, ANI needs to be changed.

\subsection{Disscusion 2}

The model is designed for high-quality processes, hence the values of $p_{0}=0.001$ and $p_{1}=0.002$ are investigated in the propesed model, as presented in Table 5 . In the previous parts, in order to elaborate on the application of model, some arbitarily values for $p_{0}$ and $p_{1}$ had been chosen to reach the optimal solution in a short and appropriate time, so that logical behavior of the model can be analyzed.

The aim of this section is to implement model in real high-quality processes. The amounts of upper and lower control limits are detemined according to the related equations, which are described in problem statement, and for different values of parameter $r$ in order to consider statistical control limits. Then, the optimal value of parameter $r$ is chosen based on objective function of economic model. First, the determined LCL and UCL are substituted in the objective function, and the value of $r$ parameter which minimizes the objective function is selected as optimal one. With regard to the applied method for determination of parameter $r$ in Section 3, the model is optimized economically, and the desired statistical standards can be satisfied simultenously. As a result, inapplicable solutions for high-quality processes in Table 3 will be revised.

As shown in Table 5, when $r$ equals 2, the objective function will have optimum value where LCL $=54$ and $\mathrm{UCL}=8876$.

\section{Conclusion}

In this paper, a detailed description of CCC control chart is examined, which is a convenient type of control chart for high-yield processes. With respect to CCC- $r$ charts, or extension of CCC ones, an economic model

Table 5. Optimal control chart design and cost parameters according to $p_{0}=0.001$ and $p_{1}=0.002$.

\begin{tabular}{|c|c|c|c|c|c|c|c|c|c|c|c|}
\hline \multicolumn{1}{|c|}{ Exp. no. } & \multicolumn{10}{|c|}{ Process and cost parameters } & Objective function \\
\hline & & $\boldsymbol{p}_{\mathbf{0}}$ & $\boldsymbol{p}_{\mathbf{1}}$ & $\boldsymbol{C}_{\text {inv }}$ & $\boldsymbol{C}_{\text {rw }}$ & $\boldsymbol{C}_{\text {rec }}$ & $\boldsymbol{\pi}$ & $\boldsymbol{N}$ & $\mathbf{L C L}$ & $\mathbf{U C L}$ & $\boldsymbol{C}_{\text {avg }}$ \\
\hline 1 & $r=1$ & 0.001 & 0.002 & 0.5 & 50 & 400 & 0.2 & 10 & 2 & 6605 & 0.104799664 \\
2 & $r=2$ & 0.001 & 0.002 & 0.5 & 50 & 400 & 0.2 & 10 & 54 & 8876 & $\mathbf{0 . 1 0 2 2 9 7 4 7 8}$ \\
3 & $r=3$ & 0.001 & 0.002 & 0.5 & 50 & 400 & 0.2 & 10 & 213 & 10823 & 0.10257987 \\
4 & $r=4$ & 0.001 & 0.002 & 0.5 & 50 & 400 & 0.2 & 10 & 467 & 12628 & 0.103110068 \\
5 & $r=5$ & 0.001 & 0.002 & 0.5 & 50 & 400 & 0.2 & 10 & 794 & 14327 & 0.103756631 \\
\hline
\end{tabular}


based on ANI is developed in order to minimize the average cost per produced item in one production cycle and optimize decision parameters of control chart. Under different situations and assumptions of all related cost and defective fraction, different cost models were established, so that the optimal design parameters and objective value of the model were obtained in each experiment. The results of comparison study show that objective function and control limit enhancement are directly related to all cost parameters changes, but the number of all items produced in investigation period and assignable causes probability has an inverse relation with objective function. In addition, with a fixed $p_{0}$ value, the upper control limit decreases as $p_{1}$ value increases. Next, it was shown that it is necessary to consider producer and consumer's risks in the economic model. Although by putting limits on ANI in the production process, the minimum average cost per item produced has been gotten larger, the parameters LCL, UCL and $r$ would be closer to their values in practical. At the end, economically and statistically optimal design of the proposed method is discussed.

\section{References}

1. Zhang, C.W., Xie, M. and Jin, T. "An improved selfstarting cumulative count of conforming chart for monitoring high-quality processes under group inspection", International Journal of Production Research, 50(23), pp. 7026-7043 (2012).

2. Bersimis, S., Koutras, M.V. and Maravelakis, P.E. "A compound control chart for monitoring and controlling high quality processes", European Journal of Operational Research, 233(3), pp. 595-603 (2014).

3. Calvin, T. "Quality control techniques for zero defects", Components, Hybrids, and Manufacturing Technology, IEEE Transactions on, 6(3), pp. 323-328 (1983).

4. Khilare, S.K. and Shirke, D.T. "The steady-state performance of cumulative count of a conforming control chart based on runs rules", Communications in Statistics-Theory and Methods, 43(15), pp. 3135-3147 (2014).

5. Chen, Y.K., Chen, C.Y. and Chiou, K.C. "Cumulative conformance count chartwith variable sampling intervals and control limits", Applied Stochastic Models in Business and Industry, 27, pp. 410-420 (2011).

6. Amiri, A. and Khosravi, R. "Estimating the change point of the cumulative count of a conforming control chart under a drift", Scientia Iranica, 19(3), pp. 856861 (2012).

7. Ahmadi Yazdi, A. and Fallahnezhad, M.S. "Comparison between count of cumulative conforming sampling plans and Dodge-Romig single sampling plan", Communications in Statistics-Theory and Methods, 46(1), pp. 189-199 (2017).
8. Ahmadi Yazdi, A. and Fallahnezhad, M.S. "An optimization model for designing acceptance sampling plan based on cumulative count of conforming run length using minimum angle method", Hacettepe Journal of Mathematics and Statistics, 44(5), pp. 1271-1281 (2015).

9. Acosta-Mejia, C.A. "Two-sided charts for monitoring nonconforming parts per million", Quality Engineering, 25(1), pp. 34-45 (2012).

10. Xie, M., Lu, X.S., Goh, T.N. and Chan, L.Y. "A quality monitoring and decision-making scheme for automated production processes", International Journal of Quality \& Reliability Management, 16(2), pp. 148157 (1999).

11. Yllmaz, Ş. and Burnak, N. "An economic approach to the management of high-quality processes", Quality and Reliability Engineering International, 29(5), pp. 681-690

12. Ohta, H., Kusukawa, E. and Rahim, A. "A CCC-r chart for high-yield processes", Quality and Reliability Engineering International, 17(6), pp. 439-446 (2001).

13. Chan, L.Y., Lai, C.D., Xie, M. and Goh, T.N. "A two-stage decision procedure for monitoring processes with low fraction nonconforming", European Journal of Operational Research, 150(2), pp. 420-436 (2003).

14. Chen, J.T. "Design of cumulative count of conforming charts for high yield processes based on average number of items inspected", International Journal of Quality \& Reliability Management, 30(9), pp. 942-957 (2013).

15. Duncan, A.J. "The economic design of $\mathrm{X}$ charts used to maintain current control of a process", Journal of the American Statistical Association, 51(274), pp. 228-242 (1956).

16. Lorenzen, T.J. and Vance, L.C. "The economic design of control charts: a unified approach", Technometrics, 28(1), pp. 3-10 (1986).

17. Mortarino, C. "Duncan's model for $\bar{X}$-control charts: sensitivity analysis to input parameters", Quality and Reliability Engineering International, 26(1), pp. 17-26 (2010).

18. Celano, G., Castagliola, P., Trovato, E. and Fichera, $\mathrm{S}$. "The economic performance of the shewhart $\mathrm{t}$ chart", Quality and Reliability Engineering International, 28(2), pp. 159-180 (2012).

19. Zhang, H.Y., Xie, M., Goh, T.N. and Shamsuzzaman, M. "Economic design of time-between-events control chart system", Computers \& Industrial Engineering, 60(4), pp. 485-492 (2011).

20. Kudo, K., Ohta, H. and Kusukawa, E. "Economic design of a dynamic CCC-r chart for high-yield processes. economic", Quality Control, 19(1), pp. 7-21 (2004).

21. Moghaddam, A.S., Amiri, A. and Bashiri, M. "Multiobjective economic-statistical design of cumulative count of conforming control chart", International Journal of Engineering-Transactions A: Basics, 27(10), pp. 1591-1600 (2014). 
22. Xie, M., Goh, T.N. and Kuralmani, V., Statistical Models and Control Charts for High-Quality Processes, Springer Science \& Business Media (2002).

23. Woodall, W.H. "Controversies and contradictions in statistical process control", Journal of Quality Technology, 32(4), pp. 341-350 (2000).

\section{Biographies}

Mohammad Saber Fallahnezhad graduated from Sharif University of Technology, Tehran, Iran, and is currently an Associate Professor of Industrial Engineering in Yazd University, Yazd, Iran. He obtained his BS, MS, and PhD degrees from Sharif University of Technology, Tehran. His research has focused on Quality Control and Engineering, Stochastic Modeling, Dynamic Programming, and Sequential Analysis. He is the recipient of both a distinguished researcher award and an outstanding lecturer award from Yazd University.

Vida Golbafian is currently an MSc student in Industrial Engineering Department of Yazd University. She obtained her BSc degree from Sadjad University, Mashhad. Her research interests have focused on Quality Control and project management. 